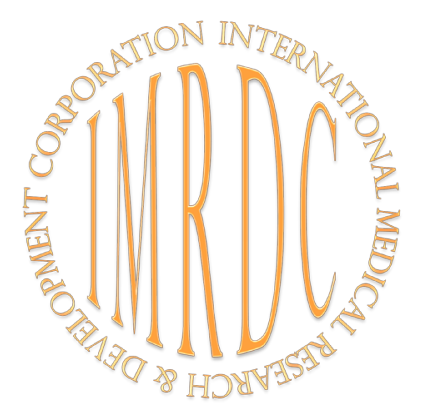

\title{
Left Ventricular Mass Appropriateness in Hypertensive Patients with Metabolic Syndrome
}

\author{
Nigora Z. Srojidinova, $\mathrm{PhD}$ \\ Republican Center of Cardiology, Tashkent, Uzbekistan
}

\begin{abstract}
The objective of this study was to evaluate the frequency of inappropriate left ventricular (LV) mass (iLVM) and factors associated with iLVM in hypertensive patients with metabolic syndrome (MetS). The study included 178 male patients (mean age $45.9 \pm 10.5 \mathrm{yrs}$ ) with hypertension (HT) and MetS. iLVM in HT patients with MetS was associated with LV diastolic dysfunction and an intensity of vascular remodeling process. Hyperinsulinemia contributes to LVM appropriateness, which aggravates the cardiovascular remodeling with development of the significant LV diastolic dysfunction and endothelium dysfunction. (Int $\mathbf{J}$ Biomed. 2016;6(3):170-173.).
\end{abstract}

Key Words: hypertension • metabolic syndrome • left ventricular hypertrophy • inappropriate left ventricular mass.

\section{Abbreviations}

BP, blood pressure; DBP, diastolic BP; EDV, end-diastolic volume; LVM, left ventricular mass; aLVM, appropriate LVM; iLVM, inappropriate LVM; LVMI, LVM index: LVH, left ventricular hypertrophy; MetS, metabolic syndrome; SBP, systolic BP.

\section{Introduction}

Left ventricular hypertrophy $(\mathrm{LVH})$ is a well-known prognostic factor for cardiovascular events. ${ }^{[1]} \mathrm{LVH}$ develops as the consequence of an increase in LV mass (LVM) secondary to chronic overload. A LV anatomical adaptation that balances cardiac load is, therefore, compensatory. However, at least in arterial hypertension (HT), a number of patients exhibit levels of LVM that exceed the need to sustain cardiac workload, a condition that has been defined as inappropriate LVM (iLVM). Inappropriateness of LV mass has been reported to be an independent prognostic factor, regardless of the presence of LVH or not. ${ }^{[2]}$ The inappropriate or excessive growth of LVM is associated with metabolic abnormality, systolic dysfunction, and LV concentric geometry, which is independent of the presence of hypertension. ${ }^{[3-5]}$ iVMI has also been reported to be associated with diastolic dysfunction, which can be demonstrated using various transmitral blood flow parameters. ${ }^{[6]}$

The objective of this study was to evaluate the frequency of iLVM and factors associated with ILVM in HT patients with MetS.

\section{Subjects and Methods}

\section{Study population}

Participants were consecutively enrolled from among outpatients in the Hypertension Department of the Republic Centre of Cardiology and written informed consent was obtained from all study participants. Exclusion criteria included a history of myocardial infarction, angina pectoris, heart failure, stroke, chronic renal insufficiency. No subjects with clinically overt diabetes were included. All procedures were approved by the Ethic Committee of the Republic Center of Cardiology.

\section{Anthropometric measurements}

SBP and DBP were measured by using a mercury blood pressure device after the subjects had rested longer than 5 min. Body mass index (BMI) was calculated by weight $(\mathrm{kg})$ divided by the squared height $(\mathrm{m})\left(\mathrm{kg} / \mathrm{m}^{2}\right)$. The waist circumference (WC) was measured in the standing position, at the level of umbilicus, located midway between the lower costal margin (bottom of lower rib) and the iliac crest (top of pelvic bone).

Biochemical analysis

After 12 hrs of fasting, blood glucose (FBG), total 
cholesterol (TC), triglyceride (TG), high-density lipoprotein cholesterol (HDL-C) and low-density lipoprotein-cholesterol (LDL-C) levels were obtained. The fasting serum insulin level was measured by immunoenzyme assay (Access ultrasensitive insulin, Beckman Coulter ${ }^{\mathrm{TM}}$ ). Standard glucose tolerance test was performed to all patients. Insulin resistance status was calculated by using the homeostatic model assessmentinsulin resistance (HOMA-IR). ${ }^{[7]}$ The calculation formula was as follows: HOMA-IR $=($ fasting insulin $[\mu \mathrm{IU} / \mathrm{mL}] \times$ fasting blood glucose $[\mathrm{mM} / \mathrm{L}]) / 22.5$. Microalbuminuria (MAU) was measured by immunoenzyme assay (RANDOX, Great Britain) and defined as an albumin urinary excretion between $20-200 \mathrm{mg} / \mathrm{ml}$.

\section{Definitions of the MetS}

According to IDF (2005), the MetS is present when the waist circumference is increased $(\mathrm{M}:>94 \mathrm{~cm} ; \mathrm{F}:>80 \mathrm{~cm}$ ) and at least two of the following factors are present: TG 1.7 $\mathrm{mmol} / \mathrm{l}(150 \mathrm{mg} / \mathrm{dl})$ or greater; low HDL-C $(\mathrm{M}<1.03 \mathrm{mmol} / \mathrm{l}$; $\mathrm{F}<1.29 \mathrm{mmol} / \mathrm{l})$; SBP greater than $130 \mathrm{mmHg}$ or DBP greater than $85 \mathrm{~mm} \mathrm{Hg}$ or treatment of previously diagnosed HT; increased fasting plasma glucose $(>5.6 \mathrm{mmol} / \mathrm{l})$ or previously diagnosed DM [8].

\section{Echocardiographic measures}

Echocardiography was performed using ultrasound system (En VisorC, PHILLIPS, Holland). Left ventricular dimension and wall thickness was measured from twodimensional guided M-mode echocardiographic tracings on the parasternal long axis view. LVM was estimated by using the Penn convention. It was indexed for body surface area to estimate LVMI. The presence of LVH was defined for LVMI $\geq$ $125 \mathrm{~g} / \mathrm{m}^{2}$ [9]. LV end-diastolic and end-systolic volumes were calculated using Teichholz's formula. ${ }^{[10]}$ Stroke volume was generated (mL/beat) and stroke work (SW in gram-meters/ beat $\left[\mathrm{g}-\mathrm{m} /\right.$ beat]) was computed ${ }^{[11]}$ as follows:

cuff systolic BP x stroke volume x 0.0144 .

LV systolic function was estimated as systolic shortening measured at the endocardial and midwall levels. ${ }^{[12]}$

Individual LVM was estimated using formula:

$$
\text { Predicted LVM }=55.37+6.64 \times \text { height }\left(\mathrm{m}^{2.7}\right)+0.64 \times \mathrm{SW}
$$$$
\text { (g-m/beat) - } 18.07 \times \text { gender }
$$

where male $=1$ and female $=2$.

Observed LVM (oLVM) was divided by predicted LVM $(\mathrm{pLVM})$ and was expressed as a percentage (oLVM/pLVM). With this method, every individual served as a reference for him/herself.

iLVM was defined as an excess of $>28 \%$ from the predicted value (ie, oLVM/pLVM $>128 \%$ ) and low LVM as a decrease of $>27 \%$ from the predicted value (ie, oLVM $/ \mathrm{pLVM}$ $<73 \%)^{[13]}$

The following parameters were measured by pulsewave Doppler: peak velocities of early (E) and late diastolic filling (A), deceleration time (DT), isovolumic relaxation time (IVRT). The ratio of early diastolic to late diastolic mitral inflow velocities was calculated (E/A).

\section{Carotid Ultrasound Imaging}

Carotid and brachial scans were obtained by highresolution B-mode ultrasound by a $7.5 \mathrm{MHz}$ linear array transducer (S4-2, PHILLIPS). Left and right common carotids were examined in antero-lateral, postero-lateral, or mediolateral directions. Longitudinal images of the distal common carotid, in which the interfaces were very clear, were obtained. Carotid intima-medial thickness (IMT) was measured in the far wall of the common carotid artery, $1 \mathrm{~cm}$ proximal to the carotid bulb in a region free of plaques.

Data were stored and analyzed with the Statistica 6.0 statistical software package. All of the data are expressed as mean $\pm \mathrm{SD}$. Characteristics of study groups are compared using Student $t$ tests or nonparametric test, as appropriate. Differences among prospectively defined subgroups were analyzed by ANOVA. $P<0.05$ was considered statistically significant.

\section{Results}

The study included 178 male patients (mean age $45.9 \pm 10.5 \mathrm{yrs}$ ) with hypertension (HT) and MetS. Among studied patients, Stage 1 HT was identified in $36.5 \%$ patients, Stage 2 HT in $37.1 \%$ patients, and Stage 3 HT in $26.4 \%$ patients. Coronary heart disease was identified in $11.8 \%$ patients, smoking in $33.7 \%$ patients. About $77.5 \%$ and $83.1 \%$ patients had LVH and LV diastolic dysfunction, respectively. The impaired EDV was identified in $83.1 \%$ patients. The baseline characteristics of patients are reported in Table 1.

\section{Table 1.}

\section{Baseline characteristics of patients}

\begin{tabular}{|l|c|}
\hline \multicolumn{1}{|c|}{ Variable } & Parameters \\
\hline BMI, kg/m² & $31.8 \pm 3.8$ \\
Waist ratio, cm & $108.6 \pm 9.8$ \\
Mean SBP, mmHg & $155.9 \pm 15.7$ \\
Mean DBP, mmHg & $99.7 \pm 9.5$ \\
Fasting glucose, mmol/l & $5.13 \pm 0.85$ \\
Postload glucose, mmol/l & $6.09 \pm 2.25$ \\
Fasting insulin, U/ml & $19.49 \pm 15.51$ \\
HOMA-IR & $4.49 \pm 3.89$ \\
TC, mg/dl & $227.90 \pm 42.82$ \\
TG, mg/dl & $229.73 \pm 146.98$ \\
HDL-C, mg/dl & $40.32 \pm 8.02$ \\
LDL-C, mg/dl & $141.48 \pm 36.10$ \\
\hline
\end{tabular}

At baseline, 43 (24.16\%) patients had aLVM, and 135 (75.84\%) patients had iLVM. It should be noted that there were no patients with ILVM with a normal LVMI. aLVM with an abnormal LVMI was identified in $6(3.37 \%)$ patients; all patients with iLVM had abnormal LVMI. (Table 2,3).

Table 2.

Characteristics of patients according to changes in LVMappropriateness

\begin{tabular}{|l|c|}
\hline \multicolumn{1}{|c|}{ Variable } & $\mathrm{n}, \%$ \\
\hline low LVM, $<73 \%$ & 0 \\
\hline aLVM, $73-128 \%$ & $43(24.6)$ \\
\hline iLVM, $>128 \%$ & $135(75.84)$ \\
\hline low- expressed iLVM, $128-155.9 \%$ & $36(20.22)$ \\
\hline moderate expressed iLVM, $156-183.9 \%$ & $50(28.09)$ \\
\hline greatly expressed iLVM, $>184 \%$ & $49(27.53)$ \\
\hline
\end{tabular}


Table 3.

Characteristics of patients according to changes in LVMappropriateness and $\mathrm{LVH}$

\begin{tabular}{|l|c|c|}
\hline \multirow{2}{*}{\multicolumn{1}{c|}{ Variable }} & \multicolumn{2}{|c|}{ LVM appropriateness } \\
\cline { 2 - 3 } & aLVM & iLVM \\
\hline without LVH, $\mathrm{n}(\%)$ & $37(20.79)$ & 0 \\
\hline with LVH, $\mathrm{n}(\%)$ & $6(3.37)$ & $135(75.84)$ \\
\hline
\end{tabular}

An analysis of clinic, demographic, and biochemical parameters, taking into account LVM appropriateness in observed patients, has detected a strict contribution of number of factors (duration of HT, SBP, DBP) to development of iLVM. Along with ILVM, HT patients with MetS were characterized by considerable changes in the serum insulin level and HOMA-IR. This determines a probable contribution of hyperinsulinemia in the iLVM development (Table 4).

Table 4.

Comparison between groups of aLVM versus iLVM

\begin{tabular}{|l|c|c|c|}
\hline \multicolumn{1}{|c|}{ Parameters } & aLVM n=43 & $P$ & $\begin{array}{c}\text { iLVM } \\
\mathrm{n}=135\end{array}$ \\
\hline Age, $\mathrm{y}$ & $41.5 \pm 9.0$ & 0.0015 & $47.3 \pm 10.6$ \\
\hline Duration of HTN, y & $3.2 \pm 2.0$ & 0.0005 & $5.7 \pm 4.5$ \\
\hline BMI, kg/m ${ }^{2}$ & $31.8 \pm 3.7$ & $N S$ & $31.8 \pm 3.9$ \\
\hline Waist ratio, cm & $106.8 \pm 8.2$ & $N S$ & $109.1 \pm 10.2$ \\
\hline Mean SBP, mmHg & $149.5 \pm 12.9$ & 0.002 & $158.0 \pm 16.0$ \\
\hline Mean DBP, mmHg & $93.2 \pm 6.2$ & 0.000000 & $101.8 \pm 9.5$ \\
\hline Observed LVM, gr & $258.3 \pm 31.6$ & 0.000000 & $348.7 \pm 72.1$ \\
\hline Index LVM, gr/m ${ }^{2}$ & $123.5 \pm 11.7$ & 0.000000 & $166.8 \pm 34.8$ \\
\hline$\Delta \mathrm{D}, \%$ & $6.73 \pm 6.21$ & 0.007 & $4.12 \pm 5.27$ \\
\hline IMT, mm & $0.76 \pm 0.23$ & 0.000000 & $0.98 \pm 0.24$ \\
\hline MAU, mg/l & $14.6 \pm 16.6$ & 0.03 & $32.87 \pm 35.53$ \\
\hline TC, mg/dl & $225.4 \pm 38.0$ & $N S$ & $228.7 \pm 44.3$ \\
\hline TG, mg/dl & $213.4 \pm 99.5$ & $N S$ & $234.9 \pm 159.1$ \\
\hline HDL-C, mg/dl & $41.4 \pm 7.9$ & $N S$ & $39.9 \pm 8.1$ \\
\hline LDL-C, mg/dl & $139.4 \pm 39.6$ & $N S$ & $142.1 \pm 35.1$ \\
\hline Fasting glucose, mmol/l & $5.32 \pm 0.40$ & $N S$ & $5.06 \pm 0.94$ \\
\hline Postload glucose, $\mathrm{mmol} / 1$ & $5.56 \pm 1.13$ & $N S$ & $6.35 \pm 2.60$ \\
\hline Fasting insulin, U/ml & $8.8 \pm 5.06$ & 0.012 & $21.27 \pm 15.97$ \\
\hline HOMA-IR & $2.15 \pm 1.40$ & 0.031 & $4.88 \pm 4.05$ \\
\hline & & & \\
\hline
\end{tabular}

Further analysis according to the degree of LVM appropriateness has shown the certain peculiarities of cardiovascular remodeling process in HT patients with MetS. A greatly expressed iLVM (>184\%) was associated with significantly higher SBP and DBP levels. Patients with greatly expressed iLVM had more expressed parameters of vascular remodeling $(\triangle \mathrm{D}$, IMT, MAU) as compared with patients with aLVM (Table 5).

\section{Discussion}

LVH detection in HT patients is very important for the risk stratification and treatment. Our study showed a high LVH prevalence among HT patients with MetS, especially iLVM. The obtained results demonstrated that the appropriateness of LVM is an independent factor determining LV diastolic dysfunction, along with age, HT duration and BP level. Some studies have demonstrated that the presence of inappropriate LVM implies a greater risk of cardiovascular events, either in the presence or in the absence of traditionally defined LVH. ${ }^{[13,14]}$ Moreover, we have detected that hyperinsulinemia and insulin resistance also contributes to LVM appropriateness.

Table 5.

Parameters of system and central hemodynamic and the endothelium dysfunction markers depending on changes in LVM appropriateness

\begin{tabular}{|l|c|c|c|c|c|}
\hline Parameters & $\begin{array}{c}73-128 \% \\
\mathrm{n}=43\end{array}$ & $\begin{array}{c}128- \\
155.9 \% \\
\mathrm{n}=36\end{array}$ & $\begin{array}{c}183.9 \% \\
\mathrm{n}=50\end{array}$ & $\begin{array}{c}>184 \% \\
\mathrm{n}=49\end{array}$ & $\mathrm{~F} / \mathrm{p}$ \\
\hline $\mathrm{SBP}, \mathrm{mmHg}$ & $149.6 \pm 12.9$ & $156.7 \pm 20.3$ & $159.4 \pm 13.9$ & $157.6 \pm 14.6$ & $\begin{array}{c}3.5 / \\
0.017\end{array}$ \\
\hline DBP, mmHg & $93.2 \pm 6.1$ & $100.3 \pm 9.1$ & $102.1 \pm 9.0$ & $102.6 \pm 10.3$ & $\begin{array}{c}10.59 / \\
0.0000\end{array}$ \\
\hline $\mathrm{SV}, \mathrm{ml}$ & $113.2 \pm 19.4$ & $101.3 \pm 28.2$ & $88.2 \pm 16.3$ & $87.3 \pm 16.3$ & $\begin{array}{c}17.03 / \\
0.0000\end{array}$ \\
\hline EF, \% & $71.0 \pm 4.0$ & $71.5 \pm 5.4$ & $67.8 \pm 5.5$ & $62.8 \pm 7.9$ & $\begin{array}{c}19.96 / \\
0.0000\end{array}$ \\
\hline E, m/sec & $0.73 \pm 0.16$ & $0.71 \pm 0.11$ & $0.68 \pm 0.14$ & $0.61 \pm 0.16$ & $\begin{array}{c}5.91 / \\
0.0007\end{array}$ \\
\hline $\mathrm{A}, \mathrm{m} / \mathrm{sec}$ & $0.67 \pm 0.15$ & $0.64 \pm 0.13$ & $0.67 \pm 0.17$ & $0.69 \pm 0.33$ & $0.41 / 0.7$ \\
\hline E/A, m/sec & $1.26 \pm 1.01$ & $1.15 \pm 0.26$ & $1.08 \pm 0.34$ & $0.95 \pm 0.23$ & $4.8 / 0.003$ \\
\hline IVRT, msec & $101.3 \pm 24.9$ & $107.7 \pm 39.1$ & $133.1 \pm 43.3$ & $147.8 \pm 46.0$ & $\begin{array}{c}13.34 / \\
0.0000\end{array}$ \\
\hline$\Delta \mathrm{D}, \%$ & $6.73 \pm 6.22$ & $6.17 \pm 4.39$ & $3.48 \pm 5.93$ & $3.25 \pm 4.82$ & $4.8 / 0.003$ \\
\hline IMT, mm & $0.76 \pm 0.23$ & $0.79 \pm 0.20$ & $1.03 \pm 0.23$ & $1.08 \pm 0.20$ & $\begin{array}{c}24.64 / \\
0.0000\end{array}$ \\
\hline MAU, mg/l & $14.60 \pm 6.64$ & $15.39 \pm 5.97$ & $22.1 \pm 14.7$ & $25.9 \pm 16.5$ & $\begin{array}{c}8.57 / \\
0.0000\end{array}$ \\
\hline
\end{tabular}

\section{Conclusion}

- iLVM in HT patients with MetS is associated with LV diastolic dysfunction and an intensity of vascular remodeling process.

- Hyperinsulinemia contributes to LVM appropriateness, which aggravates the cardiovascular remodeling with development of the significant LV diastolic dysfunction and endothelium dysfunction.

\section{Competing financial interests}

None.

\section{Materials \& Correspondence}

Dr. Nigora Srojidinova_nigora5505@gmail.com

\section{References}

1. Levy D, Garrison RJ, Savage DD, Kannel WB, Castelli WP. Prognostic implications of echocardiographically determined left ventricular mass in the Framingham Heart Study. N Engl J Med. 1990;322:1561-6.

2. de Simone G, Verdecchia P, Pede S, Gorini M, Maggioni AP. Prognosis of inappropriate left ventricular mass in 
hypertension. Hypertension. 2002;40:470-6.

3. Palmieri V, de Simone G, Roman MJ, Schwartz JE, Pickering TG, Devereux RB. Ambulatory blood pressure and metabolic abnormalities in hypertensive subjects with inappropriately high left ventricular mass. Hypertension. 1999;34:1032-40.

4. de Simone G, Pasanisi F, Contaldo F. Link of nonhemodynamic factors to hemodynamic determinants of left ventricular hypertrophy. Hypertension. 2001;38:13-8.

5. Celentano A, Palmieri V, Esposito ND, et al. Inappropriate left ventricular mass in normotensive and hypertensive patients. Am J Cardiol. 2001;87:361-3, A10.

6. de Simone G, Kitzman DW, Palmieri V, et al. Association of inappropriate left ventricular mass with systolic and diastolic dysfunction.Am J Hypertens. 2004;17:828-33..

7. Matthews DR, Hosker JP, Rudenski AS, Naylor BA, Treacher DF, Turner RC. Homeostasis model assessment: insulin resistance and beta-cell function from fasting plasma glucose and insulin concentrations in man. Diabetologia. 1985; 28:412- 419 .

8. Alberti KG, Zimmet P, Shaw J. The metabolic syndrome-a new worldwide definition. Lancet. 2005; 366:1059-1062.

9. Mancia G, de Backer G, Dominiczak A, Cifkova R, Fagard R, Germano G, et al. 2007 Guidelines for the Management of Arterial Hypertension. The Task Force for the Management of
Arterial Hypertension of the European Society of Hypertension (ESH) and of the European Society of Cardiology (ESC). J Hypertens. 2007; 25:1105-1187.

10. Teichholz LE, Kreulen T, Herman MV, Gorlin R. Problems in echocardiographic volume determinations: echocardiographic-angiographic correlations in the presence of absence of asynergy. Am J Cardiol. 1976; 37:7-11.

11. de Simone G, Devereux RB, Kimball TR, Mureddu GF, Roman MJ, Contaldo F, Daniels SR. Interaction between body size and cardiac workload: influence on left ventricular mass during body growth and adulthood. Hypertension. 1998;31:1077-1082.

12. de Simone G, Devereux RB, Roman MJ, Ganau A, Saba PS, Alderman MH, Laragh JH. Assessment of left ventricular function by the midwall fractional shortening/end-systolic stress relation in human hypertension [published erratum appears in J Am Coll Cardiol. 1994;24:844]. J Am Coll Cardiol. 1994;23:1444-1451.

13. de Simone G., Verdecchia P., Pede S., Gorini M., Maggioni A.P. Prognosis of Inappropriate Left Ventricular Mass in Hypertension. The MAVI Study. Hypertension. 2002;40:470-476. 14. de Simone G, Palmieri V, Koren M, Mensah G, Roman MJ, Devereux RB. Prognostic implications of the compensatory nature of left ventricular mass in arterial hypertension. J Hypertens. 2001;19:119-125. 